\title{
Defect Detection on Printed Fabrics Via Gabor Filter and Regular Band ${ }^{\star}$
}

\author{
Xuejuan Kang ${ }^{\mathrm{a}, *}$, Panpan Yang ${ }^{\mathrm{b}}$, Junfeng Jing ${ }^{\mathrm{b}}$

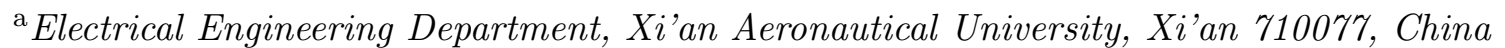 \\ ${ }^{\mathrm{b}}$ School of Electronics and Information, Xi'an Polytechnic University, Xi'an 710048, China
}

\begin{abstract}
Two methods are proposed in this paper to inspect printed fabrics. One method is to apply a genetic algorithm to select parameters of optimal Gabor filter. Optimal Gabor filter can reduce the noise information of printed fabrics, which can achieve defect detection of printed fabrics. The other is in utilizing distance matching function to determine the unit of printed fabrics. Extracting features on a moving unit of printed fabrics can realize defect segmentation of printed fabrics. Two approaches of defect detection have their own advantages. Detecting method with Gabor filter using genetic algorithm has perfect detection results of random printed fabrics, the other method based on statistical rule can receive better defect detection results of regular printed fabrics. Both methods can be realized in practice and detection time of proposed methods can occupy little in total detection time.
\end{abstract}

Keywords: Defect Detection; Gabor Filter; Regular Band; Textile Fabrics

\section{Introduction}

Defects of textile fabrics can influence selling price and resulting in the reduction of pricing by about $45 \%$ to $65 \%$ [1] of the original product. Artificial detection has a key role in real fabric detection, while artificial detection has a lower of success rate at the speed of only $15-20 \mathrm{~m} / \mathrm{min}$ [2]. Accuracy of artificial detection only can reach $60-70 \%$ [3].

Numerous algorithms are currently proposed in fabric detection. Fabric detection approaches are classified into the statistical, the model [4] and the spectral [5,6]. The statistical approach is based on texture features of fabrics and is defined as a measurement of "energy" [7] in a window of each fabric pixel. Model method applies semblable textures to match the captured textures. Cohen of Drexel University of American used Gauss Markov Random Filed (GMRF) [8] to obtain parameters of defect-free fabrics with perfect detection results, and complexity of analysis on fabrics is its fault. Spectral method is suitable for directional textured fabrics. Tsai and

${ }^{\star}$ Project supported by Scientific Research Program Funded by Natural Science Foundation of China (No. 61301276); and Shaanxi Provincial Education Department (No. 2013JK1084).

* Corresponding author.

Email address: 67807595@qq.com (Xuejuan Kang). 
Heish $[9,10]$ utilized a combination of DFT to detect directional textured images. Operating the frequency components of homogenous non-defective regions could influence frequency components of defective regions.

Genetic algorithm is a general framework of solving complex system optimization, which does not depend on specific areas of problems and has a strong robustness of problem species. Genetic algorithm is mainly used in function optimization, combinatorial optimization, scheduling problem of production, robotics and image processing and so on. Function optimization of image processing with genetic algorithm is applied in this paper. Distance matching function is used to inspect the period of fabrics in [11], but the accuracy of inspection results is low.

Gabor filter is widely used in fabric defect detection. In general, defect detection methods of Gabor filter are classified into two parts: one is in applying Gabor filters to describe channels to obtain detection results on fabrics. The other is to select optimal Gabor filter.

Ngan and Pang $[12,13]$ used a golden image as a convolution filter on testing image. The periodic waveforms of resultant matrix prove that fabric defect could be segmented. Henry Y. T. Ngan [14] proposed LBP operator of window and corresponding feature vector to detect defects of patterned fabrics in [15]. Ngan and Pang put forward regular band to complete defect detection of patterned fabrics. Period length is selected to obtain perfect detection results.

In fact, one method of this paper is building optimal Gabor filter via selecting Gabor parameters. Gabor filters are trained by non-defective fabrics in goal function, and genetic algorithm is a method to receive the minimum of goal function, thus optimal Gabor filters can be generated. Optimal Gabor filters can extract defective information of textile fabrics, thus defect segmentation can be achieved. The other method is combining regular band and distance matching function to realize defect detection of fabrics. Distance matching function is to determine the fabric unit. Regular band is to extract features of fabric unit. Proposed methods have comparisons of various fabrics. It is concluded that detecting methods of Gabor filter has better results of inspecting random textured fabrics, and regular band of proposed methods could receive perfect results in detection of patterned fabrics.

The whole paper can be classified into four sections: The first section is an introduction covering previous research of printed fabrics. The second section describes detection method of Gabor filter. The third section offers another detection method of regular band. The final section provides experimental results and resultant comparisons of proposed methods.

\section{Detection Method with Gabor Filter}

\subsection{Gabor Function}

Gabor function is an exponential function that defines the sinusoidal wave frequency $u_{0}$ and rotated orientation $\theta$, meanwhile, Gabor function is defined by modulating 2-D Gaussian function $[16,17]$. The real part of 2-D Gabor function is used as Gabor filter. Because the imaginary part demands a mass of calculations and has little influence on detection results. Gabor filter function can be formulated as Eq. (1):

$$
g_{e}(x, y)=\exp \left\{-\frac{1}{2}\left[\left(\frac{x^{\prime}}{\sigma_{x}}\right)^{2}+\left(\frac{y^{\prime}}{\lambda \sigma_{x}}\right)^{2}\right]\right\} \cos \left(2 \pi u_{0} x^{\prime}\right)
$$


among

$$
\begin{gathered}
{\left[\begin{array}{l}
x^{\prime} \\
y^{\prime}
\end{array}\right]=\left[\begin{array}{cc}
\cos \theta & -\sin \theta \\
\sin \theta & \cos \theta
\end{array}\right]\left[\begin{array}{l}
x-T_{1} \\
y-T_{2}
\end{array}\right]} \\
\sigma_{y}=\lambda \sigma_{x}
\end{gathered}
$$

Orientation $\theta$ finishes rotation from value $x$ and $y$ to the corresponding $x^{\prime}$ and $y^{\prime}$, and parameters $\left(\sigma_{x}, \sigma_{y}\right)$ represent variances of $x$ axis and $y$ axis respectively. $\lambda$ is the ratio of corresponding variances. $T_{1}$ and $T_{2}$ act as conversion parameters of $x$ axis and $y$ axis.

2-D Gabor functions can be built by rotating and zooming the basic Gabor function. The specific direction and size have strong influence on fabric detection results. The results of real part of $2-\mathrm{D}$ Gabor function are changed with $\sqrt[5]{4}$ multiples of frequency and a $\pi / 6$ rotation of angles, thus perfect performance of Gabor function can be obtained. Gabor function with adaptable parameters $u_{0}$ and $\theta$ presents various shapes. Proposed method chooses suitable shapes of various textures. It is seen that optimal Gabor filter should own the most similar envelope with pixels of non-defective fabrics. To construct an optimal Gabor filter, goal function in Eq. (2) should be reached:

$$
E=\min _{\omega, T_{x}, T_{y}, \lambda, \theta, u_{0}}\left[\sum_{x, y}\left(I M(x, y)-\omega g_{e}(x, y)\right)^{2}\right]
$$

where IM is defect-free fabrics, $g_{e}(x, y)$ means Gabor function. $\omega$ is a coefficient to harmonize the relationship. Variance ratio $\lambda$ is limited to $[1.0,2.0]$. The range of radial frequency $u_{0}$ is $[1.9$, 5.7]. Orientation usually lies within $[0, \pi]$.

\subsection{Genetic Algorithm}

Genetic algorithm [18] is a random searching method which is structured by genes, and in this paper genes are made of binary codes. Optimal or approximate solutions with higher fitness of goal function are picked out by selection, crossover and mutation operation. Finally practical problems are solved.

In the genetic algorithm, Gabor filter parameters in goal function E in Eq. (2) constitute the whole parameter individual. Genetic algorithm searches the minimum of goal function E so that the optimal Gabor parameters can be obtained. The experimental results show that the parameters obtained by genetic algorithm have to be dealt with the following procedure [19] in Eq. (3). The aim of operation is to adapt to fabric texture and end with perfect defect detection.

$$
\left\{\begin{array}{c}
\theta=\theta_{g a}-\frac{5}{6} \pi \\
u_{0}=u_{0 g a}-1.9
\end{array}\right.
$$

Parameters $\theta_{g a}$ and $u_{0 g a}$ are extracted from genetic algorithm. Orientation $\theta$ and frequency $u_{0}$ are applied in optimal Gabor filters. The other parameters keep the original in optimal Gabor filters. $I_{i}(x, y)$ is a resultant image. Asterisk $\left(^{*}\right)$ represents convolution operation. Convolution of fabrics and Gabor filter can be defined as follows:

$$
I_{i}(x, y)=\left\{\left[g_{e}(x, y) * I(x, y)\right]^{2}\right\}^{\frac{1}{2}}
$$




\subsection{Binarization in Filtered Images}

A $7 \times 7$ median filter is used for smoothing filtered fabrics. The maximum gray level value $\lambda_{\max }$ and minimum gray level value $\lambda_{\min }$ in central widow $\mathrm{W}$ of defective-free fabrics are calculated as thresholds $\lambda_{\max }$ and $\lambda_{\text {min }}$. Gray values between thresholds $\lambda_{\max }$ and $\lambda_{\min }$ in defect detection are replaced with 0 , the others are endowed with 255 . Binary resultant images $D(x, y)$ can be calculated with Eq. (5):

$$
D(x, y)=\left\{\begin{array}{rll}
255 & B(x, y)>\lambda_{\max } & \text { or } \quad B(x, y)<\lambda_{\min } \\
0 & B(x, y) \leq \lambda_{\max } & \text { or } \quad B(x, y) \geq \lambda_{\min }
\end{array}\right.
$$

\section{Detection Method Using Regular Band}

\subsection{Distance Matching Function}

Distance matching function proposed by Oh et al. [20] is defined to calculate the periodic distance of periodic function. In order to realize regular band algorithm [21], statistical pixels of fabrics must be computed with a certain length to express the features. Parameters $m$ and $n$ mean the length of regular band on column and row of patterned fabrics. Parameter $\mathrm{p}$ represents periodic distance. Fabrics could be acted as a two-dimensional function $f(x, y)$, and the results of $f(x, y)$ mean the gray value of fabrics. The row or column of fabrics can be identified as the one-dimensional function, the sum of one-dimensional row or column results in the distance matching function can be shown as Eq. (6)-(7).

$$
\begin{aligned}
& \operatorname{sum}_{\lambda x}(p)=\sum_{x=1}^{M} \sum_{y=1}^{N-p}[f(x, y)-f(x, y+p)]^{2} \\
& \operatorname{sum}_{\lambda y}(p)=\sum_{y=1}^{N} \sum_{x=1}^{M-p}[f(x, y)-f(x+p, y)]^{2}
\end{aligned}
$$

The resultant difference between variable and forward variable of distance matching function is identified as first forward difference. The difference of distance matching function with variable $p+1$ and variable $p$ is named as $D_{1}(p)$. First forward differences of distance matching function are listed in Eq. (8)-(11).

$$
\begin{gathered}
D_{1}(p)=\operatorname{sum}_{\lambda x}(p+1)-\operatorname{sum}_{\lambda x}(p) \\
D_{1}(p-1)=\operatorname{sum}_{\lambda x}(p)-\operatorname{sum}_{\lambda x}(p-1) \\
D_{2}(p)=\operatorname{sum}_{\lambda y}(p+1)-\operatorname{sum}_{\lambda y}(p) \\
D_{2}(p-1)=\operatorname{sum}_{\lambda y}(p)-\operatorname{sum}_{\lambda y}(p-1)
\end{gathered}
$$

Second forward difference $\Delta D_{1 \max }(p)$ is the difference of first forward differences with variable $p+1$ of fabrics. The maximum of second forward difference is extracted to get the counterpart periodic distance. The periodic distance of fabrics is equal to obtained periodic distance $p$.

$$
\Delta D_{1 \max }(p)=D_{1}(p)-D_{1}(p-1)
$$




$$
\Delta D_{2 \max }(p)=D_{2}(p)-D_{2}(p-1)
$$

The process of distance matching function is as follows:

Step 1: Input detecting fabrics.

Step 2: Pick a reasonable range of variable $p(p<M$ and $p<N)$. Using Eq. (6) and (7) to calculate distance matching function.

Step 3: Calculate second forward difference of distance matching function with variable $\mathrm{p}$ as Fig. 1.

Step 4: Get the maximum of second forward difference so that $p$ would be determined.

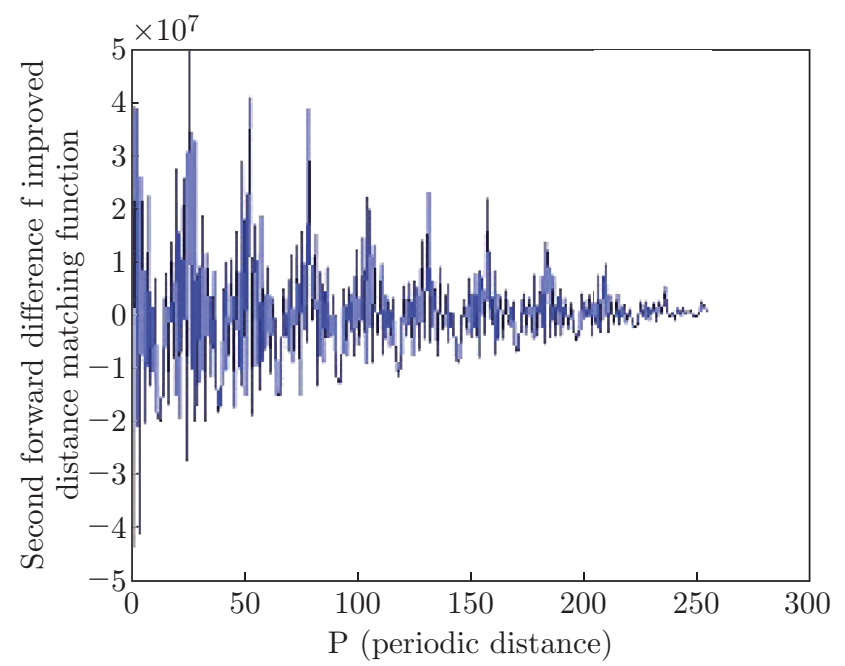

(a)

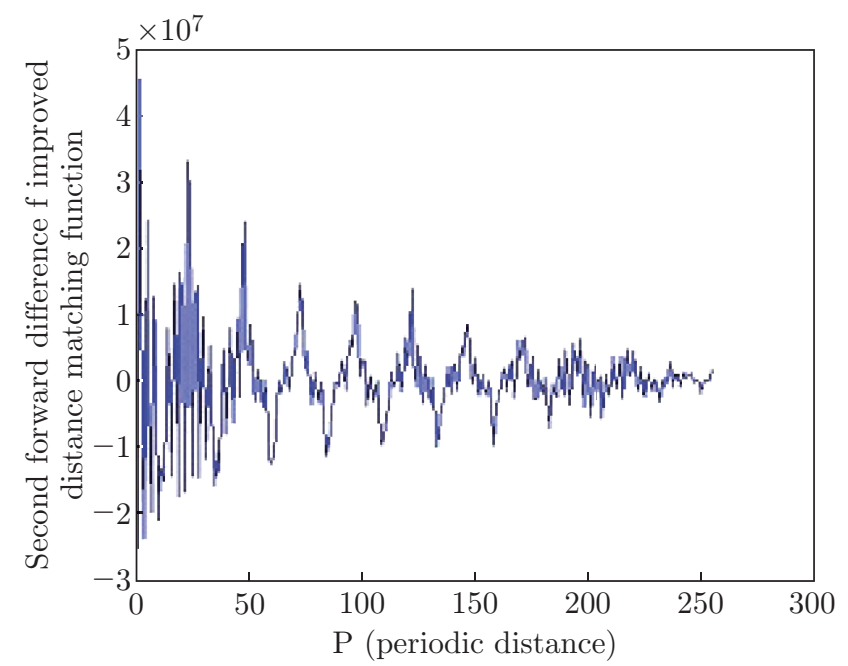

(b)

Fig. 1: Second forward difference of distance matching function on fabric rows (a) and columns (b)

The distance matching function can determine the size of patterned unit $(m, n)$, and the consequences of determining patterned unit from fabrics are exhibited in Fig. 2. The parameters $\mathrm{m}$ and $\mathrm{n}$ are listed in Table 1 . The size of patterned unit $(m, n)$ are applied in regular band to detect patterned defects.

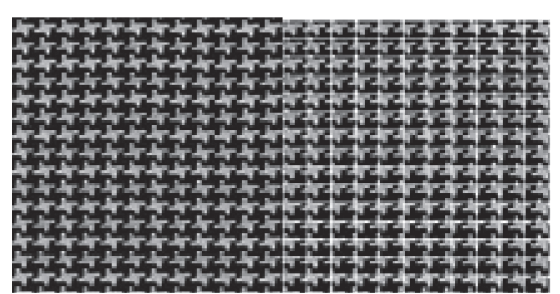

(a)

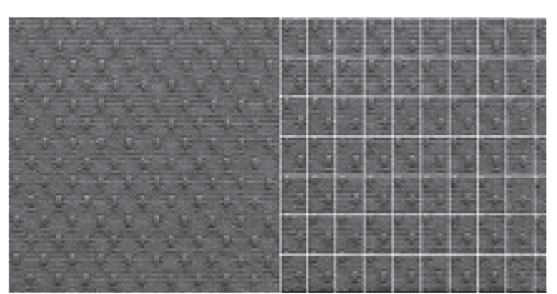

(b)

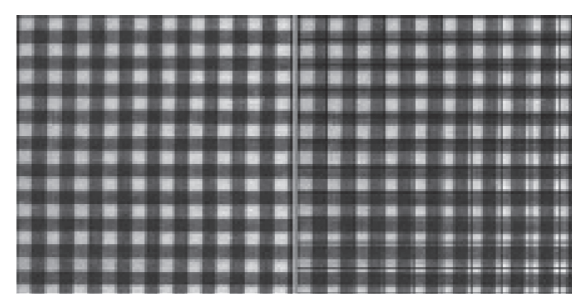

(c)

Fig. 2: Patterned unit of fabric detection

\subsection{Kernel of Regular Band}

The average value of fabric n-length pixels of training fabrics is calculated with Eq. (14). IM(x,y) represents the inspecting fabrics. Parameter $r_{1}$ means the beginning position of regular band of 
fabrics. Parameter $n$ is the length of regular band. The size of $\operatorname{IM}(x, y)$ is $(M, N)$. Parameters $x$ and $y$ are positions of fabrics. Average value $M_{0}$ is used for subtracting all pixels of fabrics.

$$
M_{0}=\left(\sum_{x=1}^{M}\left(\sum_{r_{1}=1}^{N-n+1}\left(\left(\sum_{y=r_{1}}^{r_{1}+n-1} I M(x, y)\right) / n\right) / N-n+1\right)\right) / M
$$

Feature one and feature two are helpful in the detection of patterned fabrics as Eq. (16) and (17). $I M(x, y)$ is the result of equalization operation. $S(x, y)$ shown in Eq. (11) is the result of $I M(x, y)$ by $M_{0} . N$ and $M$ are the size of inspecting fabrics. Parameters $\mathrm{n}$ and $\mathrm{m}$ are equal to the size of fabric unit via distance matching function. First feature and second feature are extracted within the moving window. Parameters $(m, 1)$ and $(n, 1)$ are the size of fabric row and fabric column. $M_{1}$ is to compute the mean of moving window of $S(x, y)$. Feature one can elongate the gap between defect pixels and non-defective pixels. Feature two in Eq. (17) depends on the difference between $S(x, y)$ and mean of corresponding $S(x, y)$ of non-defective fabrics to recognize defects of fabrics.

$$
\begin{gathered}
S(x, y)=F(x, y)-M_{0} \\
f_{1}=\sum_{x=r_{1}}^{r_{1}+n-1} S(x, y)^{3}-\sigma, \quad r_{1} \in[1, N-n+1] \\
f_{2}=\frac{\sum_{x=r_{1}}^{r_{1}+n-1}\left(S(x, y)-M_{1}\right)}{n} ; r_{1} \in[1, N-n+1]
\end{gathered}
$$

Among

$$
M_{1}=\left(\sum_{x=1}^{M}\left(\sum_{r_{1}=1}^{N-n+1}\left(\left(\sum_{y=r_{1}}^{r_{1}+n-1} S(x, y)\right) / n\right) / N-n+1\right)\right) / M
$$

Five non-defective fabrics are used in the training step. The minimum and maximum of first feature and second feature are set as the thresholds. The final thresholds should be set as a combination of row threshold and column threshold. According to the calculated threshold, Feature one and feature two are compared with the threshold of training fabrics. If the feature one and feature two of sample fabrics are included in the scope of threshold, 0 would be given the corresponding $D(x, y)$, otherwise it would be set to 255. $D(x, y)$ is defined in Eq. (19).

$$
\begin{gathered}
\left\{\begin{array}{l}
D(x, y)=255 \\
\left(f_{1}(x, y) \notin \text { Threshold or } f_{2}(x, y) \notin \text { Threshold }\right)
\end{array}\right. \\
\left\{\begin{array}{l}
D(x, y)=0 \\
\left(f_{1}(x, y) \in \text { Threshold and } f_{2}(x, y) \in \text { Threshold }\right)
\end{array}\right.
\end{gathered}
$$

\section{Experimental Results}

In the experiments, 40 training samples and 90 testing samples are applied in proposed methods. The experimental fabrics are from the TILDA database [22] and Henry Y. T. Ngan from Industrial Automation Research Laboratory Dept. of Electrical and Electronic Engineering, the University of Hong Kong. Matlab of computer is used to implement the proposed algorithms. 


\subsection{Analysis of Detection Time}

Table 1 shows the total detection time of regular band and Gabor filter. Training time and detection time are listed in Table 1 . The sum of detection time and training time equals to total running time. Training time takes the most of total run time. Fast detection time promises the possibility of operation in real situations. The complexity of devised method is $O(M N)$, which connects with fabric size. Irregular fabrics use regular band with patterned unit $(1,1)$ to inspect fabric defects. Detection time must be small to realize the real-time of industry. Proposed methods both have fast detection time, but Gabor filter needs much training time. Parameters $\mathrm{m}$ and $\mathrm{n}$ of regular band are the size of patterned unit and are extracted via distance matching function in training time.

Table 1: Detection time with proposed methods

\begin{tabular}{ccccccccc}
\hline $\begin{array}{c}\text { Images } \\
\text { of Fig. } 8\end{array}$ & $\begin{array}{c}\text { (a) Using } \\
\text { regular } \\
\text { band }\end{array}$ & $\begin{array}{c}\text { (b) Using } \\
\text { regular } \\
\text { band }\end{array}$ & $\begin{array}{c}\text { (c) Using } \\
\text { regular } \\
\text { band }\end{array}$ & $\begin{array}{c}\text { Average of } \\
\text { regular }\end{array}$ & $\begin{array}{c}\text { (a) Using } \\
\text { Gabor } \\
\text { filter }\end{array}$ & $\begin{array}{c}\text { (b) Using } \\
\text { Gabor } \\
\text { filter }\end{array}$ & $\begin{array}{c}\text { (c) Using } \\
\text { Gabor } \\
\text { filter }\end{array}$ & $\begin{array}{c}\text { Average } \\
\text { of Gabor } \\
\text { filter }\end{array}$ \\
\hline Parameter n & 23 & 27 & 27 & & & & & \\
Parameter m & 17 & 37 & 23 & & & & & \\
\hline Training time & 2.316 & 3.229 & 2.402 & 2.6496 & 61.866 & 59.873 & 59.873 & 60.537 \\
(seconds) & 981 & 209 & 773 & 543 & 072 & 685 & 685 & 8023 \\
\hline Detection & 0.204 & 0.242 & 0.223 & 0.223 & 0.203 & 0.264 & 0.237 & 0.235 \\
time (seconds) & 037 & 415 & 430 & 294 & 046 & 329 & 629 & 0013 \\
\hline
\end{tabular}

\subsection{Statistical Results of Detecting Fabrics}

There are four kinds of defect detection results: Overall Detection (OD), True Detection (TD), False Alarm (FA) and Misdetection (MD) [23]. The white area of binary image being equivalent to the corresponding defect on fabrics can explain TD. FA demonstrates that the white area includes the corresponding defect region and other white regions of fabrics. OD seems to be compounded by TD and FA. MD shows no white areas in resultant images. The fabric detection's statistical results are derived as Table 2. Table 2 includes results of regular band and Gabor filter. It can be seen that Gabor filter has more perfect results in defect detection of TILDA database, and these fabrics own random textures. Regular band method receives more ideal detection results of patterned fabrics. Defects of patterned fabrics include Hole, Thin Bar, Broken End, Netting Multiple and Thick Bar.

\subsection{Comparisons of Detection Results}

The results of Gabor filter and regular band are listed in Fig. 3, which covers box-patterned, starpatterned fabric backgrounds with Hole, Thin Bar, Broken End, Netting Multiple and Thick Bar in the experiments. The regular band has a good performance in detection of patterned fabrics. Because regular band applies the regularity of patterned fabrics, various defects can be inspected ignoring background of patterned fabrics. Gabor filter can remove background influence and 
Table 2: Statistical results of inspecting fabrics

\begin{tabular}{|c|c|c|c|c|c|c|c|c|}
\hline Detection & esults & Broken & Thin & Thick & Netting & Holl & Knots & $\begin{array}{c}\text { Fabrics from } \\
\text { TILDA }\end{array}$ \\
\hline Overall & Regular & $100 \%$ & $100 \%$ & $100 \%$ & $93 \%$ & $100 \%$ & $100 \%$ & $93 \%$ \\
\hline Detection & band & $(15 / 15)$ & $(15 / 15)$ & $(15 / 15)$ & $(15 / 15)$ & $(15 / 15)$ & $(5 / 5)$ & $(4 / 5)$ \\
\hline (OD)\% & Gabor & $33.3 \%$ & $100 \%$ & $100 \%$ & $0 \%$ & $66.7 \%$ & $100 \%$ & $100 \%$ \\
\hline & filter & $(5 / 15)$ & $(15 / 15)$ & $(15 / 15)$ & $(0 / 15)$ & $(10 / 15)$ & $(5 / 5)$ & $(5 / 5)$ \\
\hline True & Regular & $93 \%$ & $86.7 \%$ & $100 \%$ & $93 \%$ & $93 \%$ & $100 \%$ & $86 \%$ \\
\hline Detection & band & $(14 / 15)$ & $(13 / 15)$ & $(15 / 15)$ & $(14 / 15)$ & $(14 / 15)$ & $(5 / 5)$ & $(4 / 5)$ \\
\hline (TD)\% & Gabor & $33.3 \%$ & $100 \%$ & $100 \%$ & $0 \%$ & $66.7 \%$ & $100 \%$ & $100 \%$ \\
\hline & filter & $(5 / 15)$ & $(15 / 15)$ & $(15 / 15)$ & $(0 / 15)$ & $(10 / 15)$ & $(5 / 5)$ & $(5 / 5)$ \\
\hline False & Regular & $7 \%$ & $13.3 \%$ & $0 \%$ & $0 \%$ & $7 \%$ & $0 \%$ & $7 \%$ \\
\hline Alarm & band & $(1 / 15)$ & $(2 / 15)$ & $(0 / 15)$ & $(0 / 15)$ & $(1 / 15)$ & $(0 / 5)$ & $(1 / 5)$ \\
\hline & Gabor & $0 \%$ & $0 \%$ & $0 \%$ & $0 \%$ & $0 \%$ & $0 \%$ & $0 \%$ \\
\hline & filter & $(0 / 15)$ & $(0 / 15)$ & $(0 / 15)$ & $(0 / 15)$ & $(0 / 15)$ & $(0 / 5)$ & $(0 / 5)$ \\
\hline & Regular & $0 \%$ & $0 \%$ & $0 \%$ & $7 \%$ & $0 \%$ & $0 \%$ & $0 \%$ \\
\hline Misdetecti on & band & $(0 / 15)$ & $(0 / 15)$ & $(0 / 15)$ & $(1 / 15)$ & $(0 / 15)$ & $(0 / 5)$ & $(0 / 5)$ \\
\hline$(\mathrm{MD}) \%$ & Gabor & $66.7 \%$ & $0 \%$ & $0 \%$ & $100 \%$ & $33.3 \%$ & $0 \%$ & $0 \%$ \\
\hline & filter & $(10 / 15)$ & $(0 / 15)$ & $(0 / 15)$ & $(15 / 15)$ & $(5 / 15)$ & $(0 / 5)$ & $(0 / 5)$ \\
\hline
\end{tabular}

enhance defect region of fabrics. Defect region being not clear to fabric background has difficulty in being recognized with Gabor filter. Fig. 4 shows the detection results of TILDA database. Fig. 4 (a) and Fig. 4 (d) show that Gabor filter has better performance in detection of random textures. Netting Multiple Hole and Broken End of Fig. 3 exhibit the disadvantage of Gabor filter. Fabric Background being close to defect is not suitable for Gabor filter. Regular band is not fit for irregular fabrics such as Fig. 4 (a) and Fig. 4 (d).

\section{Discussion}

Two methods of fabric defect detection are proposed in this paper to achieve defect detection of patterned fabrics. Optimal Gabor filter detection algorithm is extracting whole fabric features via Gabor filtering. Proposed method of fabric defection with Gabor filter has perfect detection results. The advantage of Gabor filter is that various defects with irregular fabrics can be inspected, and the disadvantage of Gabor filter is that fabric defect must have difference with fabric background in color. Regular band is calculating fabric feature of fabric unit to realize defect detection. Regular band can receive perfect results in defect inspection of patterned fabrics. 


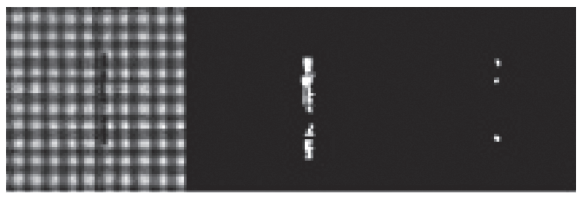

Thin bar

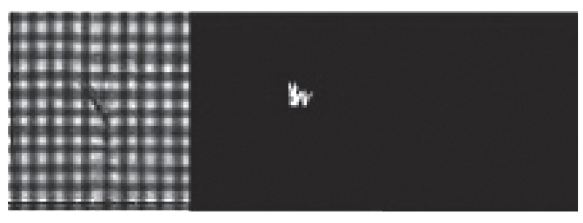

Netting multiple

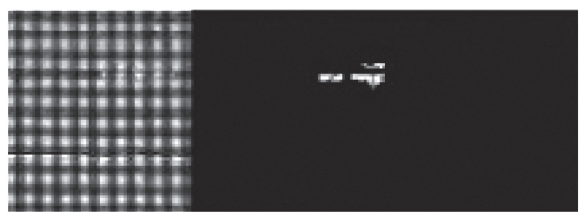

Broken end

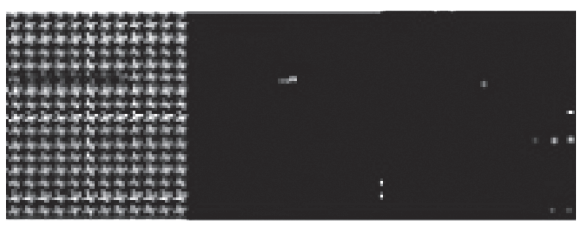

Thin bar

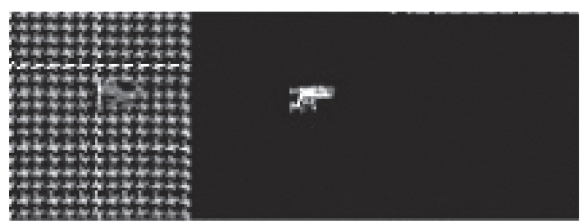

Netting multiple

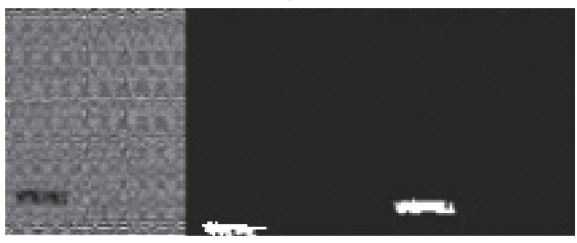

Thin bar

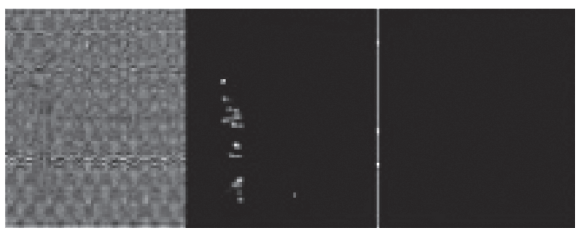

Netting multiple

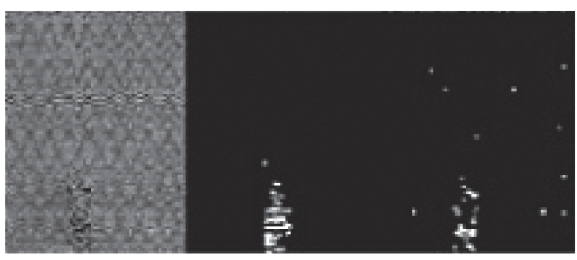

Hole

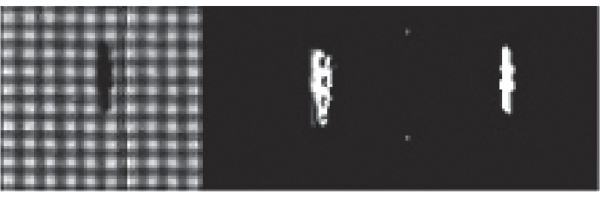

Thick bar

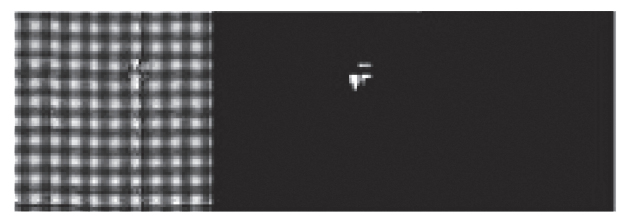

Hole

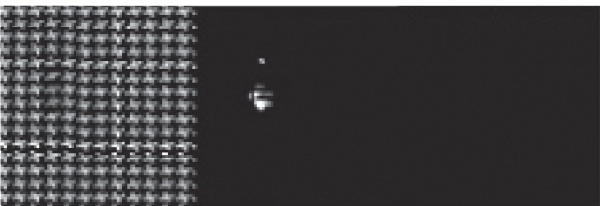

Broken end

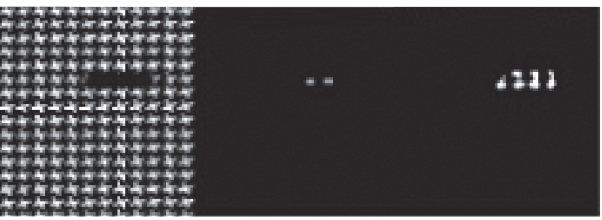

Thick bar

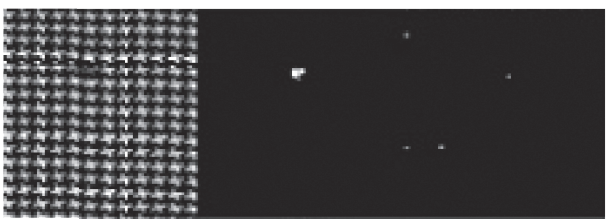

Hole

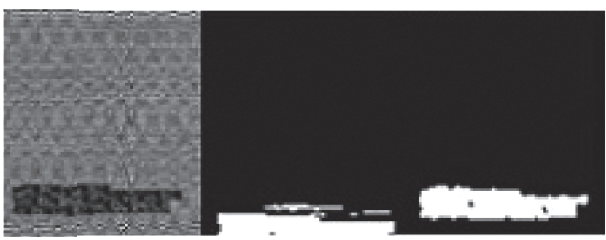

Thick bar

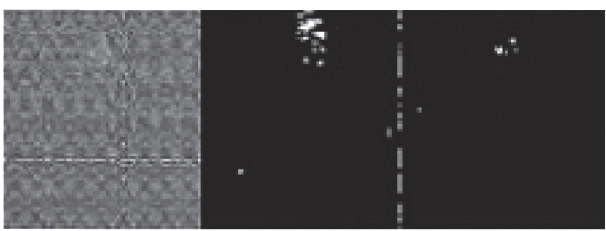

Knot

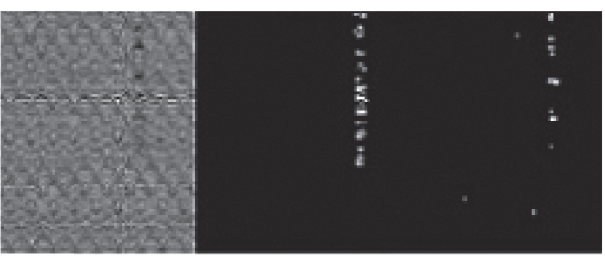

Broken end

Original fabrics regular band Gabor filter original fabrics regular band Gabor filter

Fig. 3: Detection results of patterned fabrics 

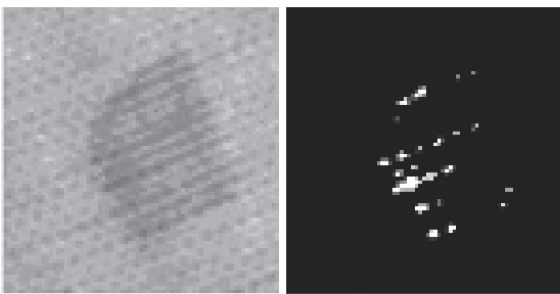

(a)
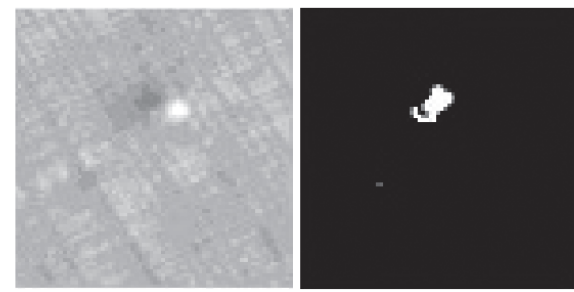

(c)
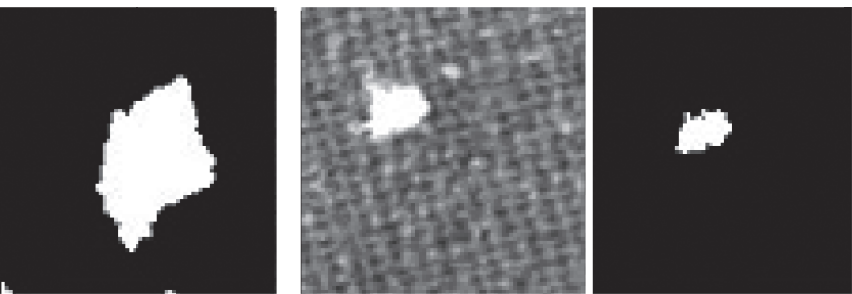

(b)
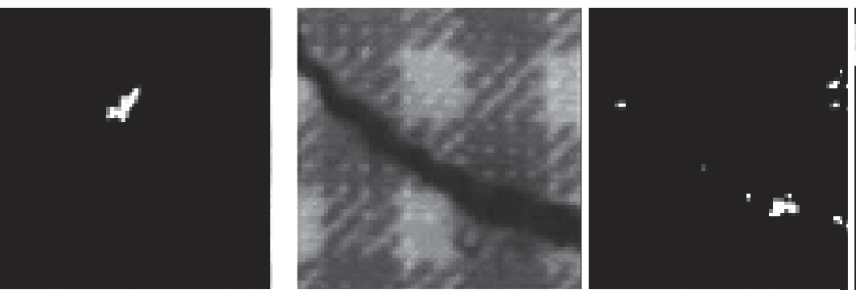

(d)
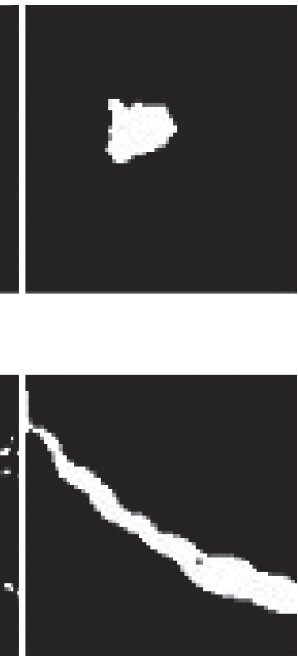

Fig. 4: Detection results of TILDA database

Proposed methods have their own advantage and disadvantage in fabric detection. Comparison has been made in this paper to conclude the advantage and disadvantage of proposed methods. Experimental results show that two proposed methods can detect fabric defects in their own condition.

The novelty of this paper can be concluded as following comparing to other researches of fabric detection [24].

1. The patterned fabrics can be detected via proposed method, and single cycle of patterned fabrics is detected automatically in this paper.

2. Training of non-defective fabrics is implemented to extract parameters or calculate segmentation threshold, thus achieving fabric detection and training step can avoid complicate calculations in detection step, so detection time can be small to realize real-time defect detection.

\section{Conclusion}

This paper proposes two approaches to detect printed fabrics. Gabor filter uses genetic algorithm to construct the optimal Gabor filter. Goal function is the standard of selecting Gabor parameters in genetic algorithm. Optimal Gabor filter can remove noise of fabric background and enhance defect information, thus defect segmentation can be finished. However regular band extracts features of fabric unit, and the size of fabric unit is determined by distance matching function in which second forward difference of fabrics is calculated to determine the patterned unit. Comparisons have been made of two proposed methods. Gabor filter has better performance in detection of irregular fabrics which defect region has difference with background fabrics. Regular band has advantage in defect detection of patterned fabrics. Theory of regular band is judging the regularity of fabrics, and defects destroy the regularity of fabrics, thus defects can be recognized. Proposed methods are implemented fast which the regularity can satisfy the real-time of practice. 


\section{References}

[1] K. L. Mak, P. Peng, Detecting defects in textile fabrics with optimal Gabor filters, International Journal of Computer Science, 1(4), 2006, 274-282

[2] Junjie Chen, Chunping Xie, Automatic cloth inspection system, Textile Science and Technology Progress, (5), 2004, 56-57

[3] Junfeng Jing, Huanhuan Zhang, Pengfei Li, Jinyuan Jia, Fabric defect detection using Gabor filters and defect classification based on LBP and Tamura method, Journal of The Textile Institute, 104(1), 2013, 18-27

[4] Amit Rawal, Prasad Potluri, Colin Steele, Prediction of yarn paths in braided structures formed on a square pyramid, Journal of Industrial Textiles, 36(3), 2007, 221-226

[5] Ajay Kumar, Computer vision-based fabric defect detection: A survey, IEEE Transactions on Industrial Electronics, 55(1), 2008, 348-363

[6] Henry Y. T. Ngan, Grantham K. H. Pang, Nelson H. C. Yung, Automated fabric defect detection - A review, Image and Vision Computing, 29(7), 2011, 442-458

[7] Xin Li, Zengpu Xu, Based on image energy fabric defect detection method, Computer Measurement and Control, 16(9), 2008, 1243-1245

[8] B. S. Manjunath, R. Chellappa, Unsupervised texture segmentation using Markov Random Filed Models, IEEE Transactions on Pattern Analysis, 13(5), 1991, 478-482

[9] Du-Ming Tsai, Shin-Min Chao, An anisotropic diffusion-based defect detection for sputtered surfaces with inhomogeneous textures, Image and Vision Computing, 23(3), 2005, 325-338

[10] Du-Ming Tsai, Chih-Chia Kuo, Defect detection in inhomogeneously textured sputtered surfaces using 3D Fourier image reconstruction, Machine Vision and Applications, 18(6), 2007, 383-400

[11] G. Oh, S. Lee, Yong Shin S., Fast determination of textural periodicity using distance matching function, Pattern Recognition Letters, 20(2), 1999, 191-197

[12] K. A. Jain, F. Farrokhnia, Unsupervised texture segmentation using Gabor filters, Pattern Recognition, 24(12), 1991, 14-19

[13] Jianwei Yang, Lifeng Liu, Tianzi Jiang, Yong Fan, A modified Gabor filter design method for fingerprint image enhancement, Pattern Recognition Letters, 24(12), 2003, 1805-1817

[14] Junfeng Jing, Hang Li, Pengfei Li, Combined fabric defects detection approach and quadtree decomposition, Journal of Industrial Textiles, 41(4), 2012, 331-344

[15] F. Tajeripour, E. Kabir, A. Sheikhi, Defect detection in patterned fabrics using modified local binary patterns, Conference on Computational Intelligence and Multimedia Applications, 2, 2007, 261-267

[16] J. Kula, M. Tunak, A. Linka, Inspection system of fabric based on texture segmentation utilizing Gabor filters, In 7th International Conference - TEXSCI 2010, Liberec, Czech Republic, 2010, September 6-8

[17] M. George Haley, Rotation invariant texture classification using modified Gabor filters, Image Processing, 1, 1995, 262-265

[18] Stephanie Forrest, Genetic algorithms: Principles of natural selection applied to computation, Science, 261(5123), 1993, 872-878

[19] K. L. Mak, P. Peng, An automated inspection system for textile fabrics based on Gabor filters, Robotics and Computer-Integrate Manufacturing, 24(3), 2008, 359-369

[20] G. Oh, S. Lee, Yong Shin S., Fast determination of textural periodicity using distance matching function, Pattern Recognition Letters, 20(2), 1999, 191-197 
[21] R. O. K. Reddy, B. E. Reddy, E. K. Reddy, Texture analysis and defect classification for fabric images using regular bands and quadratic programming, Proceedings of International Conference on Advances in Computing, Springer India, 2012, 927-934

[22] Workgroup on Texture Analysis of DFG, TILDA Textile Texture database, August 1996, http://lmb. informatik.uni-freiburg.de/research/dfg-texture/tilda

[23] Kai-Ling Mak, Pai Peng, Fabric defect detection using multi-level tuned-matched Gabor filters, Journal of Industrial and Management Optimization, 8(2), 2012, 325-334

[24] Pengfei Li, Yang Jiao, Junfeng Jing, Jiangnan Li, The high-speed fabric defect detection algorithm based on the image layered model, Journal of Fiber Bioengineering \& Informatics, 6(2), 2013, 161173 